Pulsars : Problems \& Progress

ASP Conference Series, Vol. 105, 1996

S. Johnston, M. A. Walker and M. Bailes, eds.

\title{
Energy Transport in a Rotation-Modulated Pulsar Wind
}

\author{
A. Melatos
}

Theoretical Astrophysics, Mail Code 130-33, California Institute of Technology, Pasadena CA 91125 USA

\section{B. Melrose}

Research Centre for Theoretical Astrophysics, University of Sydney, Sydney NSW 2006 Australia

\begin{abstract}
The structure and energetics of a rotation-modulated pulsar wind are examined. It is shown that the displacement current in the wind asymptotically dominates the conduction current, creating an outer radiation zone whose inner boundary lies well inside the wind termination shock. A self-consistent nonlinear-plasma-wave model of the radiation zone predicts that the ratio of Poynting flux to kinetic-energy flux at the termination shock is small $\left(\sim 10^{-3}\right.$ for the Crab), in agreement with independent observational estimates.
\end{abstract}

\section{Introduction}

The relativistic wind of a rotation-powered pulsar is usually modelled as a steady-state, ideal-magnetohydrodynamic (MHD) outflow (Michel 1969; Kennel \& Coroniti 1984; Begelman \& Li 1994, and references therein). This description is incomplete for two reasons. First, the wind is not steady in general: rotation of the star drives strong oscillating currents in the wind zone if the star's spin and magnetic axes are misaligned (Usov 1975, 1994; Coroniti 1990; Melatos \& Melrose 1996). Second, the ratio $\sigma$ of Poynting flux to kinetic-energy flux is roughly constant throughout a steady-state, ideal-MHD wind, implying $\sigma_{\mathrm{s}} \gtrsim 1$ at the termination shock if the flow is injected with $\sigma \gtrsim 1$ as many magnetospheric models predict. This is inconsistent with the value $\sigma_{\mathrm{S}} \sim 10^{-3}$ derived for the Crab pulsar from studies of pressure confinement of the wind by the Crab nebula (Kennel \& Coroniti 1984) and non-thermal particle acceleration at the termination shock (Hoshino et al. 1992). Although attempts have been made to explain this inconsistency by invoking conversion of Poynting flux to kinetic-energy flux within the wind, either via magnetic reconnection (Coroniti 1990) or a 'magnetic nozzle' effect (Begelman \& Li 1994), a complete resolution of the paradox does not yet exist (Melatos \& Melrose 1996).

In this paper, we argue that breakdown of the steady-state approximation (and, under certain circumstances, the ideal-MHD approximation) creates a radiation zone in the outer pulsar wind. A nonlinear-plasma-wave model of the radiation zone correctly predicts $\sigma_{\mathrm{s}} \ll 1$ for the Crab. 


\section{Beyond the Steady State}

In steady-state wind theories, the Maxwell displacement current is zero by fiat. This does not reflect the situation in a real rotation-modulated wind, where the displacement current exceeds the conduction current beyond a critical distance $r_{\mathrm{CR}}$ from the pulsar (Usov 1975, 1994; Melatos \& Melrose 1996). We estimate $r_{\mathrm{CR}}$ as follows. The displacement current at a distance $r$ from the pulsar has magnitude $J_{\mathrm{D}}(r) \approx \varepsilon_{0} \omega E(r)$, where $\omega$ is the stellar rotation frequency and $E(r)$ is the characteristic electric field strength at $r$. Assuming a split-monopole geometry for the wind (a good approximation in the equatorial plane far from the light cylinder $\left.r_{\mathrm{L}}=c / \omega\right)$, we can write $E(r) \approx c B_{\mathrm{L}} r_{\mathrm{L}} / r$, where $B_{\mathrm{L}}$ is the magnetic field strength at $r_{\mathrm{L}}$ (Michel 1969; Coroniti 1990). On the other hand, the conduction current at a distance $r$ has magnitude $J(r)<n(r) e c$, where $n(r)$ is the aggregate number density of electrons and positrons measured in the lab frame. (We consider a pair plasma for simplicity.) For a split-monopole wind, we can write $n(r) \approx \mathcal{N} / 4 \pi r^{2} c$, where $\mathcal{N}$ is the injection rate of electron-positron pairs. The ratio of the currents is then

$$
\frac{J_{\mathrm{D}}(r)}{J(r)} \gtrsim 1.9 \times 10^{-2}\left(\frac{\mathcal{N}}{10^{38} \mathrm{~s}^{-1}}\right)^{-1}\left(\frac{\omega}{1 \mathrm{rad} \mathrm{s}^{-1}}\right)^{-1} \frac{B_{\mathrm{L}}}{10^{6} \mathrm{G}} \frac{r}{r_{\mathrm{L}}} .
$$

It is clear from (1) that there exists a critical distance $r_{\mathrm{CR}}$ such that $J_{\mathrm{D}}(r)$ exceeds $J(r)$ for $r>r_{\mathrm{CR}}$. Typical Crab pulsar parameters $\left(\mathcal{N}=1 \times 10^{38} \mathrm{~s}^{-1}\right.$, $B_{\mathrm{L}}=1 \times 10^{6} \mathrm{G}$ ) yield $r_{\mathrm{CR}} \approx 1 \times 10^{5} r_{\mathrm{L}}$, much smaller than the distance to the termination shock $\left(r_{\mathrm{s}}=2 \times 10^{9} r_{\mathrm{L}}\right)$.

If the wind contains equal proper densities of electrons and positrons, the conduction current satisfies the more restrictive condition

$$
J(r) / n(r) e c<\left[\Gamma(r)^{2} \sin ^{2} \chi(r)+\cos ^{2} \chi(r)\right]^{-1 / 2},
$$

where $\Gamma(r)$ is the bulk Lorentz factor of the flow and $\chi(r)$ is the angle between the bulk velocity $\mathbf{U}$ and conduction current $\mathbf{J}$ in the lab frame (Melatos \& Melrose 1996). Equation (2) corresponds to the bulk-frame condition $\tilde{J}<\tilde{n} e c$; physically, $\tilde{n}$ and the component of $\tilde{\mathbf{J}}$ parallel to $\mathbf{U}$ decrease by a factor $\Gamma$ when boosting from the bulk frame to the lab frame, while the perpendicular component of $\mathbf{J}$ remains invariant. In a relativistic split-monopole wind, one has $\Gamma(r) \approx \Gamma$ (a constant) and $\chi(r) \gg \Gamma^{-1}$ for $r \geq r_{\mathrm{L}}$. The ratio $J_{\mathrm{D}}(r) / J(r)$ is therefore reduced by a factor $\Gamma$ from the value (1). Taking $\Gamma=4 \times 10^{6}$ for the Crab (Gallant \& Arons 1994), we find $J_{\mathrm{D}}(r) \gg J(r)$ everywhere outside the light cylinder.

The ideal-MHD approximation can also break down asymptotically in a rotation-modulated wind, because the plasma is not a perfect conductor at low densities (the amount of free charge available is insufficient to short out the bulk-frame electric field). In these circumstances, one must replace the 'fluxfreezing' condition $\mathbf{E}=-\mathbf{U} \times \mathbf{B}$ with a generalised, relativistic Ohm's law (Melatos \& Melrose 1996). However, ideal MHD does not break down in the region $r_{\mathrm{CR}}<r<r_{\mathrm{S}}$ for typical pulsar parameters, so the effect is only important when it sets in at $r<r_{\mathrm{CR}}$. 


\section{Asymptotic Radiation Zone}

What is the structure of the asymptotic wind zone $r>r_{\mathrm{CR}}$ ? One possibility is that the wind in this region is a large-scale, nonlinear plasma wave which selfconsistently accelerates electrons and positrons to relativistic speeds and whose electromagnetic fields approximately satisfy the source-free Maxwell equations - i.e. a radiation zone. Since the phase velocity of the nonlinear wave is very close to $c$, its wavelength $r_{\mathrm{L}} / 2 \pi$ is many times smaller than the characteristic distance over which its properties vary due to the sphericity of the outflow (except near the light cylinder). We thus regard the wave as locally planar and describe its evolution with $r$ using the WKB approximation.

A variety of planar nonlinear wave modes exist in a cold pair plasma (Kennel \& Pellat 1976, and references therein). Following Melatos \& Melrose (1996), we consider a transverse, circularly polarised wave with zero ambient magnetic field and with equal proper densities of electrons and positrons streaming relativistically in the direction of wave propagation (as well as oscillating as part of the wave itself). This mode obeys the dispersion relation

$$
\beta^{2}=1-\frac{\omega_{0}^{2}}{\Gamma \omega^{2}\left(1+\nu^{2}\right)^{1 / 2}},
$$

where $\beta$ is the refractive index, $\omega$ is the wave frequency, $\Gamma$ is the streaming Lorentz factor, $\omega_{0}=\left(n e^{2} / m_{e} \varepsilon_{0}\right)^{1 / 2}$ is an effective plasma frequency, and $\nu=$ $e E / m_{e} c \omega$ is a dimensionless wave amplitude. The mode also has the properties $J_{\mathrm{D}} / J=\left(1-\beta^{2}\right)^{-1}$ and $|\mathbf{E}+\mathbf{U} \times \mathbf{B}| /|\mathbf{E}|=1-\beta U / c$. Consequently, for $r \gg r_{\mathrm{CR}}$ $(\beta \approx 1)$ and $U \approx c$, we obtain $J_{\mathrm{D}} \gg J$ and $\mathbf{E}+\mathbf{U} \times \mathbf{B} \approx 0$ (cf. $\S 2$ ).

The WKB approximation requires that the phase-averaged particle flux and phase-averaged total energy flux (Poynting plus kinetic) in the wave be constant at all $r$. Letting $L$ be the spin-down luminosity, we therefore write (Melatos \& Melrose 1996)

$$
\begin{gathered}
\omega_{0}^{2}=\frac{\mathcal{N} e^{2} \omega^{2}}{4 \pi \varepsilon_{0} m_{e} c^{3}}\left(\frac{U}{c}\right)^{-1}\left(\frac{r}{r_{\mathrm{L}}}\right)^{-2} \\
\frac{L U}{\mathcal{N} m_{e} c^{3}}=\frac{\beta \omega^{2} \nu^{2}}{\omega_{0}^{2}}+\frac{\Gamma U}{c}\left[1-\frac{\Gamma^{2} \omega^{4}\left(1-\beta^{2}\right)^{2} \nu^{2}}{\omega_{0}^{4}}\right]^{-1 / 2} .
\end{gathered}
$$

Observations of the Crab reveal that at least $\sim 20$ per cent of the pulsar's spindown luminosity is deposited in the nebula, where it emerges reprocessed as synchrotron radiation, so the dissipationless wind considered here is a reasonable description.

\section{Poynting Flux versus Kinetic-Energy Flux}

Treating $\omega, \Gamma, \mathcal{N}$ and $L$ as fixed, equations (3), (4) and (5) can be solved for $\beta, \omega_{0}$ and $\nu$ as functions of $r$, allowing the ratio $\sigma(r)$ of Poynting flux to kinetic-energy flux to be calculated. For $\mathcal{N}$ and $\Gamma$ in their expected ranges, this calculation yields $\sigma_{\mathrm{s}} \equiv \sigma\left(r_{\mathrm{s}}\right) \ll 1$ for the Crab pulsar (see figure 1 of Melatos \& Melrose 1996), in broad agreement with independent estimates (Kennel \& Coroniti 1984; Hoshino et al. 1992) and in contrast to steady-state analyses. Furthermore, 
recent studies of non-thermal particle acceleration at the termination shock of the Crab pulsar wind appear to require $\mathcal{N} \approx 1 \times 10^{38} \mathrm{~s}^{-1}$ and $\Gamma \approx 4 \times 10^{6}$ in order to reproduce the post-shock nebular synchrotron spectrum (Hoshino et al. 1992) and the spacing and brightness of the optical wisps (Gallant \& Arons 1994). With these 'state-of-the-art' values, the nonlinear-plasma-wave model predicts $\sigma_{\mathrm{s}} \approx 3 \times 10^{-3}$, exactly the value deduced independently by Kennel \& Coroniti (1984) from the observed interaction of the wind with the surrounding remnant.

The above results are based on the transverse, circularly polarised wave mode with dispersion relation (3). Although this mode affords an excellent description of the wind energetics at $r_{\mathrm{S}}$, it does not propagate in the region $r \lesssim 1 \times 10^{5} r_{\mathrm{L}}$ and therefore yields no information about the value of $\sigma$ at $r_{\mathrm{L}}$ (Melatos \& Melrose 1996). (At $r \approx 1 \times 10^{5} r_{\mathrm{L}}$, we have $\beta=0$, and the Poynting flux vanishes.) To rectify this, we have examined more general modes to determine whether they can propagate throughout the wind and yield $\sigma_{\mathrm{s}} \ll 1$ while conserving particle flux and total energy flux. The results of this work will be reported in full elsewhere (Melatos 1996, in preparation). Here, we simply state that mixed longitudinal-transverse modes are found to be unacceptable for the same reason as the mode considered in this paper, but a transverse, circularly polarised wave with a non-zero ambient magnetic field in the direction of propagation does satisfy the above requirements. Interestingly, the latter mode leads to $\sigma_{\mathrm{L}} \equiv \sigma\left(r_{\mathrm{L}}\right) \ll 1$ for a pure pair plasma, contrary to many magnetospheric theories, but permits $\sigma_{\mathrm{L}} \sim 1$ under certain conditions if a dilute (though energetically significant) ion fraction is added (cf. Hoshino et al. 1992).

Acknowledgments. This research was supported by an Australian Postgraduate Award, NASA Grant NAG5-2756, and NSF Grant AST-93-15455. In addition, AM gratefully acknowledges receipt of an International Travel Grant from the American Astronomical Society.

\section{References}

Begelman, M. C., \& Li, Z.-Y. 1994, ApJ, 426, 269

Coroniti, F. V. 1990, ApJ, 349, 538

Gallant, Y. A., \& Arons, J. 1994, ApJ, 435, 230

Hoshino, M., Arons, J., Gallant, Y. A., \& Langdon, A. B. 1992, ApJ, 390, 454

Kennel, C. F., \& Coroniti, F. V. 1984, ApJ, 283, 694

Kennel, C. F., \& Pellat, R. 1976, J. Plasma Phys., 15, 335

Melatos, A., \& Melrose, D. B. 1996, MNRAS, in press

Michel, F. C. 1969, ApJ, 158, 727

Usov, V. V. 1975, Ap\&SS, 32, 375

Usov, V. V. 1994, MNRAS, 267, 1035 